\title{
LAVER 莱薇尔身体美白产品关于美白临床效果观察
}

\section{Clinical Observation on Whitening Effect of Laver Body Whitening Products}

\author{
周颖 \\ Ying Zhou \\ 北京思辨中医药研究院长沙分院 中国・湖南 长沙 410016 \\ Changsha branch of Beijing Institute of SiBian Chinese Medicine, Changsha, Hunan, 410016, China
}

摘要: 采用人体试验结合仪器测量的方法证明 LAVER 美白沐浴膏和 LAVER 身体美白滋养亳具有显著的全身美白效果。 应用漫反射光谱法和 FotoFinderDermoscope 仪器检测 LAVER 身体美白套装使用前后皮肤颜色的变化。使用四周后与使用前 相比, $\mathrm{L}$ 值均有增大, 且差异显著 $(\mathrm{L}=1.79 \pm 1.33, P<0.01)$ 。实验结果表明, LAVER 美白沐浴膏和 LAVER 身体美白滋养膏的美 白效果明显, 有使皮肤变白的趋势。

\begin{abstract}
It is proved that LAVER whitening bath cream and LAVER Body whitening and nourishing cream have significant whitening effect on the whole body by human body test and instrument measurement. Diffuse reflectance spectroscopy and FotoFinder Dermoscope were used to detect the changes of skin color before and after laver body whitening suit was used. After four weeks of use, $L$ value increased compared with that before use, and the difference was significant $(\mathrm{L}=1.79 \pm 1.33, P<0.01)$. The results showed that LAVER whitening bath cream and LAVER body whitening and nourishing cream had obvious whitening effect, and had the tendency of whitening skin.
\end{abstract}

关键词: 沐浴膏; 身体美白; 美白膏;人体试验; 美白效果测试

Keywords : bath cream; body whitening; whitening cream; human body test; whitening effect test

DOI : $10.36012 /$ pmr.v2i3.2296

\section{1 引言}

近年来,皮肤美白更加专注于如何去黑色素。LAVER 美 白沐浴膏和 LAVER 身体美白滋养膏利用生物活性褪黑科技 能够通过小分子精亳快速渗透肌肤底层, 直接作用于黑色素 将黑色素快速分解。本文在其研究基础上, 采用人体试验结 合仪器检测的方法来证明 LAVER 美白沐浴膏和 LAVER 身 体美白滋养膏具有显著的美白功效。

\section{2 实验测试}

\section{1 实验理论}

采用漫反射光谱法，针对皮肤颜色的特征性发色基因， 如黑色素、血红蛋白等,选择特定波长扫描, 测定皮肤表面的 反射光谱, 从而对皮肤中的血红蛋白和黑色素等进行定量, 其测量参数能较好地从生理学上解释皮肤颜色的改变 ${ }^{[1]}$ 。

\section{2 人体试验受试对象及分组}

某大学在校学生 40 名, 年龄 18 24 岁, 皮肤健康, 无化 妆品过敏史。分为四组, 每组 10 人。第一组, 使用完整成品 LAVER 美白沐浴膏和 LAVER 身体美白滋养膏; 第二组, 使 用单瓶 LAVER 美白沐浴膏; 第三组, 使用普通身体孚L作为阳 性对照组; 第四组, 使用普通沐浴露, 作为阴性对照组。实验 过程中受试者及数据采集者均不知晓分组情况。

受试者连续使用试验品 4 周, 每天晚间依照不同的分组 使用不同的试验品沐浴按摩, 作为对照。受试者左、右前臂臂 弯处, 连续测试 6 次, 取平均值, (先测右臂再测左臂); 使用 化妆品前 1 周, 以及使用 1 周、 2 周、 3 周、4 周后。

\section{3 检测方法}

利用由摄像机和显微镜头组成的 FoToFinder dermoscope 仪器作为测试仪器, 可以对色素沉着区进行拍照、放大, 并进 
行图像处理, 计算各种参数如皮损大小、边缘情况、结构参 数, 同时作出安全评价。

\section{4 检测指标}

左右前臂色差 $\mathrm{L}$ 为正值, 表示左前臂较右前臂偏白, $\mathrm{L}$ 为负值, 表示左臂较右臂偏黑。

\section{5 统计分析}

应用 SPSS 12.0 统计分析软件, 对受试者左右前臂色差 $\mathrm{L}$ 进行分析。同一实验品使用前后的参数的初始值和终末值 是否存在显著性差异, 显著性检验水平为 $P \leqslant 0.05$ 。

\section{3 测验结果}

在 Lab 的色度系统中, 色度系统表明了颜色在色空间的 位置, 并加以量化, 使颜色变化以数值的形式表达, 肉眼不能 看见的细微变化也可反映出来 ${ }^{[2]}$ 。经配对 $t$ 检验统计分析结 果如表 1 所示, LAVER 美白沐浴膏和 LAVER 身体美白滋养 膏、及单瓶 LAVER 美白沐浴露在使用过程中, 随着使用时间 的增加, L 值均发生差异性变大。表明 LAVER 美白沐浴亳和 LAVER 身体美白滋养膏有美白效果，并随着使用时间的增 加美白效果越明显。

表 1 使用后与使用前相比各组的 $L$ 值变化 (平均值士标准偏差)

\begin{tabular}{c|c|c|c|c}
\hline & 1 周后 & 2 周后 & 3 周后 & 4 周后 \\
\hline 第一组 & $0.46 \pm 1.56$ & $0.99 \pm 1.95$ & $1.42 \pm 1.27 * *$ & $1.79 \pm 1.33^{* *}$ \\
\hline 第二组 & $0.44 \pm 0.27 *$ & $0.60 \pm 0.64 *$ & $1.06 \pm 0.90 * *$ & $1.32 \pm 0.76 * *$ \\
\hline 第三组 & $0.009 \pm 0.098$ & $0.016 \pm 0.24 *$ & $0.013 \pm 0.2$ & $-0.01 \pm 0.16^{*}$ \\
\hline 第四组 & $0.007 \pm 0.097$ & $0.077 \pm 0.16$ & $0.043 \pm 0.14$ & $-0.006 \pm 0.12$ \\
\hline
\end{tabular}

注: $* \mathrm{P}<0.05, * * \mathrm{P}<0.01$ 第一组, LAVER 美白沐浴膏和 LAVER 身体美白滋养膏; 第二组, 单瓶 LAVER 美白沐浴露; 第三组, 阳性对 照;第四组,阴性对照。

使用 4 周后, LAVER 美白沐浴膏和 LAVER 身体美白滋 养膏 $(\mathrm{L}=1.79 \pm 1.33, P=0.008)$ 、单瓶 LAVER 美白沐浴露 $(\mathrm{L}=$
$1.32 \pm 0.76, P=0.008)$ 、阳性对照组（ $\mathrm{L}=-0.01 \pm 0.16, P=0.037)$ 的 $\mathrm{L}$ 值与阴性对照的 L 值对比发现, 美白效果应是: LAVER 美 白沐浴膏和 LAVER 身体美白滋养膏>单瓶 LAVER 美白沐 浴露>阳性对照组>阴性对照组。其中 LAVER 美白沐浴膏和 LAVER 身体美白滋养膏美白效果更加显著，白度效果是阳 性对照组,以及阴性对照组的两倍。同时证明 LAVER 美白沐 浴亳和 LAVER 身体美白滋养亳含有的高浓缩活性小分子对 酪氨酸酶有明显的抑制作用, 且抑制效果, 与实验结论一致。

\section{4 结论}

本文采用人体试验和仪器测量相结合的方法测试了 LAVER 美白沐浴膏和 LAVER 身体美白滋养膏的美白效果。 结果表明其活性因子成分会直接作用于黑色素并直接快速 分解黑色素, 通过 FoToFinder dermoscope 仪器的光学测试与 分析,证明其能够使黑色素在短时间内消失, 并加速消失的 速度。同时有过人体实验的角度, 其不含不刺激成分, 受试者 体验效果极佳。

\section{5 分析}

经过多次人体实验与光学仪器 FoToFinder dermoscope 的测试与分析,得出 LAVER 美白沐浴膏和 LAVER 身体美白 滋养亳具有显著的美白效果。其成分中含有的活性因子, 能 够作用于细胞中的黑色素, 深度渗透至皮肤基底层, 完美快 速分解黑色素, 达到生理学上完整的美白效果, 具有创新的 医学美容价值。

\section{参考文献}

[1] 陈卓仪,刘晓英,郑雅婷,等.皮肤黑色素形成机理与干预机制[J]. 日用化学工业,2019,49(2):113-117+125.

[2] 李玲,苏瑾,李竹,等.采用 Lab 色度系统评价某种美白化妆品的 美白功效[J].环境与职业医学,2003(1):28-30. 